LETTER TO THE EDITOR

\title{
Pomegranate Juice is Useful for the Management of Hypertension and the Improvement of Cardiovascular Health
}

\author{
Konstantinos Tziomalos ${ }^{1}$, Michael Doumas ${ }^{2,3}$ and Vasilios G. Athyros ${ }^{2, *}$ \\ ${ }^{I}$ Department of Internal Medicine, Medical School, Aristotle University of Thessaloniki, AHEPA Hospital, Thessaloniki, \\ Greece; ${ }^{2}$ Department of Internal Medicine, Medical School, Aristotle University of Thessaloniki, Hippokration Hospital, \\ Thessaloniki, Greece; ${ }^{3}$ Veteran Administration Medical Center and George Washington University, Washington, DC, \\ USA
}

Keywords: Pomegranate juice, usefulness, availability, arterial hypertension, ideal cardiovascular health.

\section{DEAR EDITOR}

We thank Prof. Liberopoulos and Dr Barkas you for their letter [1], which helps to enhance the understanding of the message of the associated Editorial.

Price and availability of pomegranate juice (PJ) are indeed two major issues. The daily cost of PJ consumption is ranging from 0.5 to $1 \$$ per day, according to the dose used. PJ is available in almost all Western and several Asian countries. PJ is preserved in deep refrigeration and is readily available during the entire year. PJ circulates mainly in two forms: pure juice in 200 to $500 \mathrm{~mL}$ bottles (adulteration is practically impossible, because the color and the taste are unique) and in $1 \mathrm{~L}$ bottles, blended with purple grape juice without added sugar. The later contains resveratrol, a type of natural phenol and a phytoalexin, found in the skin of red grapes with definite antidiabetic effects and proposed but not proved yet anti-aging and anticancer effects. Resveratrol treatment has shown beneficial effects on glucose and lipid metabolism in some, but not all studies [2,3]. Study population, resveratrol source, and dose vary widely, potentially explaining inconsistency of findings among studies. Enhancement in endothelial function, systolic blood pressure, and markers of oxidative stress and inflammation in several studies have been reported $[2,3]$.

The quantity and calorie intake is not a problem. Most studies (performed by Prof Aviram team) used a $50 \mathrm{~mL}$ PJ intake [4], mainly from the "wonderful" variety [5]. The juice has a $10 \%$ content of sugar $(5 \mathrm{~g} / \mathrm{d})$. Thus, calorie intake is not significant. In regard to diabetes mellitus the use of PJ, especially if this is mixed with purple crape juice, substantially reduces blood glucose and the risk of diabetes, and not the other way around [2-5]. As a matter of fact PJ

\footnotetext{
*Address correspondence to this author at the Department of Internal Medicine, Medical School, Aristotle University of Thessaloniki, Hippokration Hospital, Thessaloniki, Greece. 15 Marmara St, Thessaloniki, 55132, Greece; Tel: +30 2310 892606; Fax: +30 2310 835955;

E-mails: athyros@med.auth.gr and vathyros@gmail.com
}

has more antiatherogenic effects on patients with diabetes than in non-diabetic individuals [4-6].

The studies showing a beneficial effect of PJ on cardiovascular risk factors indeed included a small number of participants, were mostly not double blinded and evaluated only surrogate end-points. This is why we suggest that PJ might be only used as an adjunctive therapy for arterial hypertension (HTN) on top of other non-pharmacological interventions or drug therapy [7], and mainly in patients with HTN and high oxidative burden such as this caused by diabetes, obesity, metabolic syndrome or smoking [7]. This is not a first line therapy and is not suggested for monotherapy [7]. It is unfortunate that up to day there have been no sponsors or grants available to clear this issue once and for all, regardless of the findings, with a large scale, prospective, randomized, controlled survival study in humans.

The effect of PJ on blood pressure is reported by some studies to be as high as a $21 \%$ reduction in systolic blood pressure, which is rather sizable reduction [8].

The long term (3-years) effects of PJ on a surrogate endpoint (carotid atherosclerosis) were shown in a clinical study [8]. There were no adverse effects as reported with other antioxidants, such as alpha-tocopherol and beta-carotene [8]. Finally, PJ was shown to have the highest antioxidant potential than any other functional food or supplementary treatment [9].

Common sense suggests that all appropriate pharmacological or non-pharmacological interventions should be implemented for the optimal control of HTN, however, the use of PJ (the other parts of the fruit have no antioxidant actions) could be considered as an adjunctive therapy to improve the control of HTN and the related, mainly cardiovascular, adverse events.

\section{CONFLICT OF INTEREST}

The author(s) confirm that this article content has no conflicts of interest. 


\section{ACKNOWLEDGEMENT}

Declared none.

\section{REFERENCES}

[1] Barkas F, Liberopoulos E. Is pomegranate juice for the management of hypertension and the improvement of cardiovascular health readily available and useful? Open Hypertens J 2013 [in press].

[2] Pollack RM, Crandall JP. Resveratrol: Therapeutic Potential for Improving Cardiometabolic Health. Am J Hypertens 2013 [Epub ahead of print]

[3] Tomé-Carneiro J, Larrosa M, González-Sarrías A, Tomás-Barberán FA, García-Conesa MT, Espín JC. Resveratrol and clinical trials: the crossroad from in vitro studies to human evidence. Curr Pharm Des 2013; 19: 6064-93.

[4] Rosenblat M, Hayek T, Aviram M. Anti-oxidative effects of pomegranate juice (PJ) consumption by diabetic patients on serum and on macrophages. Atherosclerosis 2006; 187: 363-71.

Received: September 21, 2013

Accepted: September 21, 2013

(C) Tziomalos et al.; Licensee Bentham Open.
[5] Rock W, Rosenblat M, Miller-Lotan R, Levy AP, Elias M, Aviram $\mathrm{M}$. Consumption of wonderful variety pomegranate juice and extract by diabetic patients increases paraoxonase 1 association with high-density lipoprotein and stimulates its catalytic activities. J Agric Food Chem 2008; 56: 8704-13.

[6] Fuhrman B, Volkova N, Aviram M. Pomegranate juice polyphenols increase recombinant paraoxonase-1 binding to highdensity lipoprotein: studies in vitro and in diabetic patients. Nutrition 2010; 26: 359-66.

[7] Tziomalos K, Doumas M, Athyros VG. No-pharmacological Intervention: Pomegranate juice for the treatment of arterial hypertension and the improvement of cardiovascular health status. Open Hypertens J 2013 [in press].

[8] Aviram M, Rosenblat M, Gaitini D, et al. Pomegranate juice consumption for 3 years by patients with carotid artery stenosis reduces common carotid intima-media thickness, blood pressure and LDL oxidation. Clin Nutr 2004; 23: 423-33.

[9] Seeram NP, Aviram M, Zhang Y, et al. Comparison of antioxidant potency of commonly consumed polyphenol-rich beverages in the United States. J Agric Food Chem 2008; 56: 1415-22.

This is an open access article licensed under the terms of the Creative Commons Attribution Non-Commercial License (http://creativecommons.org/licenses/by-nc/3.0/) which permits unrestricted, non-commercial use, distribution and reproduction in any medium, provided the work is properly cited. 